\title{
Experimenteller Prüfaufbau zur Ermittlung von erweiterten GNSS-Empfänger-Qualitätsmerkmalen
}

\author{
Dirk Spiegel, Dr.-Ing. Uwe Becker \\ Institut für Verkehrssicherheit und Automatisierungstechnik | TU Braunschweig \\ Hermann-Blenk-Straße 42, 38108 Braunschweig, Deutschland
}

\begin{abstract}
Kurzfassung
Für das hochautomatisierte bis vollautomatische Fahren hat das Wissen über die eigene Position und die zugehörige Genauigkeit einen sicherheitsrelevanten Stellenwert. Zur Eigenlokalisation haben sich satellitenbasierte Ortungssysteme etabliert. Um derartige Systeme für den Bodenverkehr zuzulassen, bedarf es der Qualitätsermittlung der einzelnen Komponenten. Dieser Beitrag befasst sich mit der Kernkomponente des satellitenbasierten Ortungssystems, dem GNSS-Empfänger. Die Herausforderung bei der Qualifizierung von GNSS-Empfängern besteht in der Sicherstellung der Reproduzierbarkeit von Prüfszenarien und in der Berücksichtigung des stochastischen Verhaltens von GNSS-Empfängern. In diesem Beitrag werden zuerst gängige Qualifizierungsmerkmale vorgestellt und um die Merkmale Positionsaktualität und Wiederholqualität erweitert. Anschließend wird ein Prüfaufbau dargestellt, der es ermöglicht das stochastische Verhalten der GNSS-Empfänger bei instationären Messbedingungen reproduzierbar zu prüfen. Folgend wird die Durchführung von exemplarischen Messungen in Braunschweig, die Auswertung der Messfahrten und die Interpretation der Positionsaktualität und Wiederholqualität beschrieben.
\end{abstract}

Keywords: GNSS-Empfänger, Test, Zertifizierung, Wiederholgenauigkeit, Positionsaktualität

\section{Einleitung}

Für das hochautomatisierte bis vollautomatische Fahren hat das Wissen über die eigene Position und die dazugehörige Genauigkeit einen sicherheitsrelevanten Stellenwert. Die Eigenpositionierung im kinematischen Kontext ist mit satellitenbasierter Ortung vielversprechend. Satellitenbasierte Ortungssysteme bestehen in der Regel aus einem Beschleunigungssensor, einem GNSS-Empfänger und gegebenenfalls aus zusätzlichen Sensoren. Um derartige Systeme für den Bodenverkehr zuzulassen, bedarf es der Qualifizierung der einzelnen Komponenten. Eine der größten Herausforderungen bei der Qualifizierung von satellitenbasierten Ortungssystemen sind die Reproduzierbarkeit von Prüfszenarien und die eindeutige Ermittlung der Qualitätsparameter. Mittlerweile ist die Standardisierung von satellitenbasierten Ortungssystemen in den verschiedensten Gremien angekommen [3, 6, 7, 8, 11]. Diese Standards berücksichtigen jedoch nicht das stochastische Verhalten von GNSSEmpfängern. Folglich sind die errechneten Qualitätsmerkmale nur begrenzt aussagekräftig.

\section{Qualitätsmerkmale von satellite- basierten Ortungssystemen}

Die Qualifizierung von satellitenbasierten Ortungssystemen wird derzeit auf den unterschiedlichsten Ebenen durchgeführt. In der Wissenschaft haben sich drei Hauptebenen formiert. Die erste Ebene beschreibt die Eigenschaften der GNSS-Empfänger Komponenten. Dies sind beispielsweise die Qualität von Signalverstärkern, Analog-Digitalwandlern oder Quarzuhren. Die zweite Ebene umfasst die gemessenen Rohdaten. Dies sind bspw. die Pseudodistanzen zwischen Antennenzentrum und Satellit oder das Signal-zu-Rausch Verhalten. Auf der dritten Ebene wird das Endprodukt von GNSS-Empfängern untersucht. Das sind die Merkmale, die für den Anwender von Bedeutung sind.

Bei der Qualifizierung von satellitenbasierten Ortungssystemen ist die Genauigkeit, ausgedrückt durch Messrichtigkeit und -präzision in einem definierten Koordinatensystem, das Hauptmerkmal [17]. Das zu wählende Koordinatensystem ist dabei von dem Anwendungsziel abhängig. Gängig ist es die Genauigkeit in Nord und OstRichtung sowie quer und lateral zur Fahrtrichtung anzugeben. Als weitere Qualitätsmerkmale sind die allgemeine Verfügbarkeit oder die Kon- 


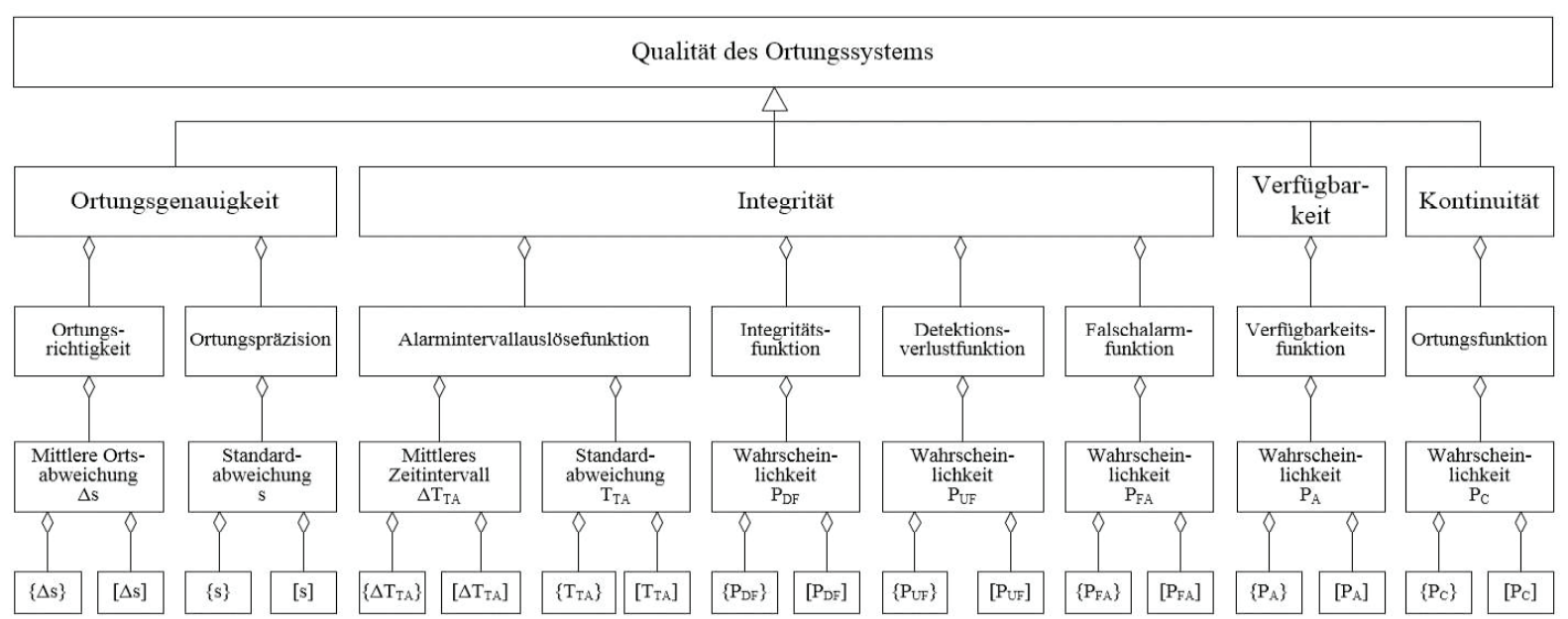

Abb. 1: Gütekriterien in UML-Klassendiagramm-Darstellung zur Beschreibung der Qualität von Ortungssystemen in Anlehnung an $[2,17]$

tinuität während domänenspezifischer Szenarien von großer Bedeutung. Die Qualität satellitenbasierter Ortungssysteme wird in diesem Beitrag im Stil der Attributhierarchie nach [13] dargestellt. Bei der Darstellung in Attributhierarchieform wird ein abstrakter Begriff durch Eigenschaften (qualitativ) und Merkmale (quantitativ) bis auf das Niveau der Messgröße und der Einheit verfeinert. Die Termini Eigenschaften und Merkmale werden dabei im Sinn des Standards DIN 2342 [4] angewandt. Die Qualität von satellitenbasierten Ortungssystemen wird in Abb. 1 dargestellt. Hier wird beispielsweise die Qualität des Ortungssystems durch die Ortungsgenauigkeit beschriebenen. Die Ortungsgenauigkeit wiederum gliedert sich in die Ortungsrichtigkeit und -präzision, die jeweils durch die mittlere Ortsabweichung und die dazugehörige Standardabweichung ausgedrückt werden. Typischerweise werden satellitenbasierte Ortungssysteme auf die Genauigkeit und Verfügbarkeit anhand einer oder sehr weniger Testfahrten bewertet. Dieses Prüfen ist jedoch nicht ausreichend. Für das Hoch- bis vollautomatisierte Fahren sind weitere Qualitätsmerkmale von großer Bedeutung. In diesem Beitrag werden zwei weitere Qualitätsmerkmale vorgestellt, die aufgrund des herausfordernden Messaufbaus nicht ermittelt werden.

\subsection{Positionsaktualität}

Bei der Eigenlokalisation im dynamischen Kontext spielt der Zeitpunkt der Bereitstellung der Positionsdaten eine wichtige Rolle. Werden die Positionsdaten zu spät bereitgestellt hat das Fahrzeug die Position zum Zeitpunkt der Messung verlassen und die gelieferten Positionsdaten sind mit steigenden Abweichungen belastet. Folglich ist die Positionsaktualität der Daten ein
Qualitätsmerkmal von satellitenbasierten Ortungssystemen. Die Positionsaktualität für einen einzelnen Messwert wird bestimmt durch ein Zeitintervall, das aus der Differenz zwischen dem Zeitpunkt an dem der Messwert an der Benutzerschnittstelle bereit steht und dem Zeitpunkt an dem die Messung ausgelöst wurde (externer Trigger oder interne Einstellungen) besteht. Die Positionsaktualität spiegelt somit die zeitliche Verzögerung der Positionsinformationen wider. Der Einfluss der Positionsaktualität ist sinnbildlich in Abb. 2 dargestellt.

Die Positionsaktualität berechnet sich nach:

$$
\begin{aligned}
t_{\text {Positionsaktualität }} & =t_{\text {Bereitstellung }}-t_{\text {Trigger }} \\
& -\Delta t_{\text {Schnittstelle }}
\end{aligned}
$$

und die aus der Positionsaktualität resultierende Positionsabweichung kann vektoriell nach:

$$
\Delta \vec{y}_{\text {Latenz }}=\vec{y}_{\text {Ref }}\left(t_{\text {Bereitstellung }}\right)-\vec{y}_{G P S}\left(t_{\text {Trigger }}\right)
$$

berechnet werden. Für Messreihen konnte gezeigt werden, dass die Positionsaktualität für große Stichproben annähernd der Normalverteilung folgen. Folglich bietet es sich an die Positionsaktualität mit dem Mittelwert und der dazugehörige Standardabweichung zu detaillieren.

\section{Wiederholgenauigkeit}

GNSS-Empfänger arbeiten nicht deterministisch. So haben Messungen auf einer Achterbahn im Heidepark Soltau gezeigt, dass GNSSEmpfänger gleicher Bauart, mit gleicher Konfiguration und mit gleichen Eingangssignalen gespeist, unterschiedliche Positionen berechnen [15]. Dies ist u. A. auf adaptive Filterparameter 


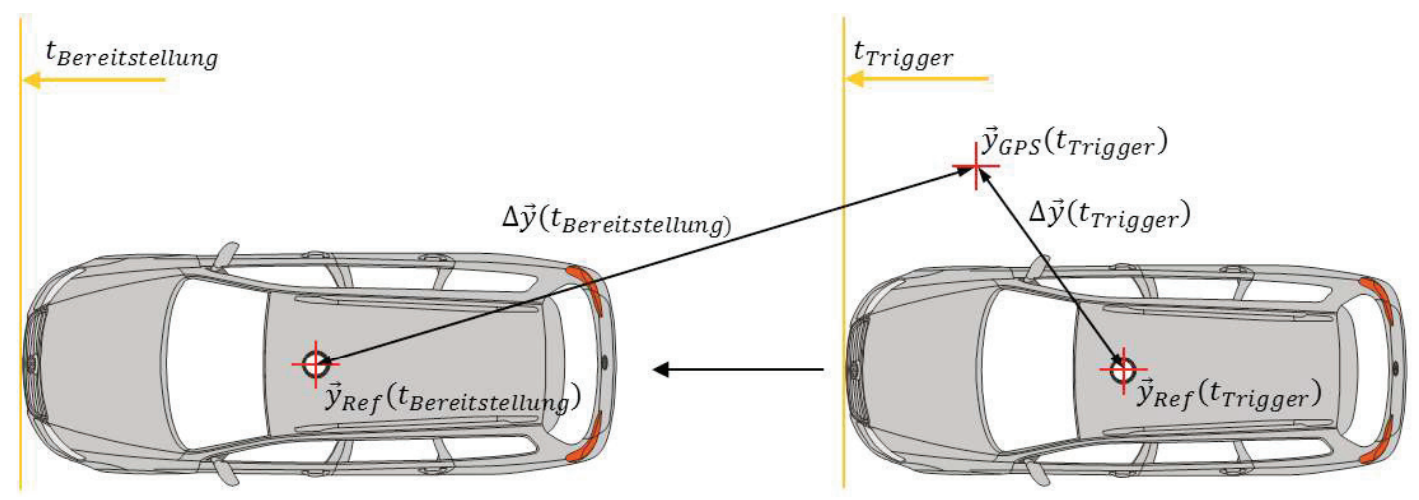

Abb. 2: Exemplarische Darstellung der Positionsaktualität

und Einflüsse wie z. B. die Temperatur einzelner Mikrokomponenten zurückzuführen. Für die angeschlossenen Systeme wäre ein deterministisches System von Vorteil. Folglich ist die Wiederholgenauigkeit ein Qualitätsparameter. Wiederholgenauigkeit kann durch das Merkmal der Wiederholstreuung quantifiziert werden. Als Maß der Wiederholstreuung wird in [15] die Varianz gewählt. Diese ist für als Maß für die Anwendung im Bodenverkehr nicht sonderlich anschaulich. Daher wird für diesen Anwendungsfall die Wurzel der Wiederholvarianz also die Wiederholstandardabweichung eines bestimmten Merkmals bestimmt. Die Wiederholstandardabweichung $\mathrm{Sr}$ berechnet sich nach [5]:

$$
s_{r}=\sqrt{\frac{\sum_{i=1}^{p}\left(n_{i j}-1\right) \cdot s_{i j}^{2}}{\sum_{i=1}^{p}\left(n_{i j}-1\right)}}
$$

mit $\mathbf{S}^{2}$ ij der Varianz des gewählten Qualitätsparameters j, der wiederholten Abspielung i und der Stichprobengröße $n_{i j}$ sowie der gesamten Anzahl von Wiederholungen p. Die Wiederholstandardabweichung kann für bestimmte Prüfszenarien bestimmt werden. Aus Gründen der Übersicht wurde hierfür kein weiterer Index in Gleichung 3 eingeführt. Damit die obige Gleichung gültig ist und der zentrale Grenzwertsatz erfüllt ist muss die Stichprobe vorher auf Ausreißer untersucht werden. Dafür wurde der Ausreißertest nach Grubbs ausgewählt. Eine genaue Beschreibung des Verfahrens findet sich in [5]. Für das Signifikanzniveau wurde der gängige Wert von 0.05 gewählt. Die maximale Anzahl zu eliminierenden Ausreißer wurde in [9] mit 2/9 spezifiziert. Warum diese Grenze gewählt wurde ist nicht näher bekannt.

\section{Prüfaufbau}

Um die in Kapitel 0 spezifizierten Qualitätsmerkmale zu bestimmen wurde ein neuer Prüfaufbau entwickelt. Dieser Prüfaufbau setzt sich aus vier Subsystemen zusammen, die mit den gleichen Satellitensignalen über einen Antennensplitter versorgt werden. So wird sichergestellt, dass sowohl das Referenzmesssystem und die Prüfgegenstände die Positionen für das gleiche Antennenzentrum berechnen und die GNSS-Empfänger den gleichen Messbedingungen unterliegen. Das erste Subsystem besteht aus den Prüfgegenständen. Für dieses Experiment wurde auf einen High-End-GNSS-Empfänger und einen Low-Cost-Empfänger zurückgegriffen. Beide Empfänger wurden so konfiguriert, dass Daten des EGNOS-Dienstes genutzt werden und die internen Filter für das Autofahren parametrisiert sind.

Das zweite Subsystem stellt die Referenz bereit. Dafür wurde ein satellitengestütztes INS-System ausgewählt. Das OxTS RT 3002 wird permanent über eine mobile Datenschnittstelle mit Echtzeitkorrekturdaten für eine virtuelle Basisstation von Axio-Net betrieben. Über die virtuelle Basisstation und das Profil der Prüfszenarien ist es möglich die Basislinie auf maximal 20 km zu begrenzen. Sowohl der Provider der Satellitenkorrekturdaten als auch der Hersteller der Referenzmesssystems spezifizieren die Genauigkeit ihrer Systeme auf $\pm 2 \mathrm{~cm}[1,12]$. Da zu erwarten ist, dass die Messabweichungen des Prüfgegenstands deutlich größer als die Messunsicherheit des Referenzmesssystems sind, wird in dieser Arbeit die Referenzposition als der wahre Wert angenommen.

Das dritte Subsystem stellt eine hochgenaue Referenz für die Bestimmung der Positionsaktualität bereit. Zur Bestimmung der Positionsaktualität ist ein hochgenaues Zeitnormal notwendig, das den Zeitpunkt des Triggerns und die Daten des Prüfgegenstands mit einem Zeitstempel versieht. Dies wurde mit dem Echtzeitsystem dSPACE MicroAutoBox realisiert. Das System 


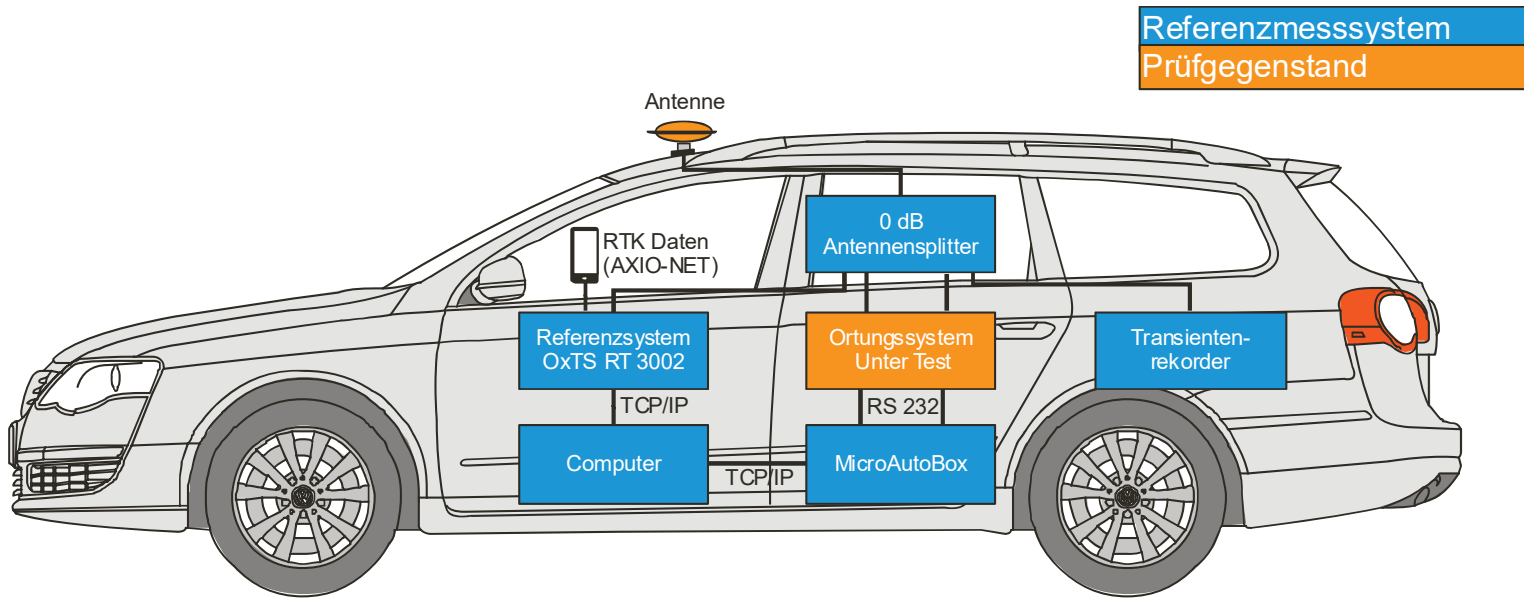

Abb. 3: Prüfaufbau zuverlässiger Qualitätsermittlung

arbeitet mit einer Taktfrequenz von $1000 \mathrm{~Hz}$. Der Zeitpunkt des Triggerns wird in diesem Versuchsaufbau über das PPS Signal von zwei verschiedenen Empfängern abgegriffen. Mit einer Genauigkeit im Nanosekundenbereich und nur sehr kurze Positionsaktualitäten durch die digitalen I/Os wird das PPS Signal als wahrer Wert angenommen. Die Positionsinformationen werden anhand des \$GPRMC Formats extrahiert. Die untersuchten Prüfgegenstände stellen diese Informationen über eine RS232-Schnittstelle bereit.

Das vierte Subsystem zur Bestimmung der Wiederholgenauigkeit ist ein Transientenrekorder. Der Transientenrekorder wandelt die Frequenzbänder, in denen GNSS-Signale auftreten, in digitale Signale um und speichert diese auf einer Festplatte. Um die Signale wieder abzuspielen, wird der Prozess umgekehrt. Somit lassen sich die aufzeichneten Signale beliebig oft wiederholt abspielen. Für dieses Experiment wurde auf ein Spirent GSS6425 Record und Playback System zurückgegriffen [16]. Der Prüfaufbau ist exemplarisch in Abb. 3 dargestellt.

\section{Prüfszenarien}

Die größten Einflüsse auf die Qualität von GNSS-Empfängern in Bodennähe sind:

- Signalschwächungen bedingt durch absorbierende Schichten,

- Mehrwegeeffekte an Materialien mit geringem Absorptionsgrad und

- komplette Signalabschattungen.

Diese Eigenschaften wurden für ausgewählte Straßen in Braunschweig und Umgebung qualitativ erhoben und in 5 Stufen unterteilt [14]. Basierend auf den qualitativ kategorisierten Straßen wurden anschließend 5 Szenarien (Groß- stadtfahrt, Stadtfahrt, Überlandfahrt, Autobahnfahrt, Waldfahrt) erstellt. Das Szenario Großstadtfahrt ist dabei wiederum in Unterszenarien aufgeteilt. Es wird unterschieden in Häuserschlucht mit/ohne Blätter tragende(n) Bäumen. Die Gesamtlänge des Prüfszenarios beträgt ca. $78 \mathrm{~km}$ lang und kann unter normalen Verkehrsbedingungen in ca. $1 \mathrm{~h} 40$ min durchfahren werden. Dies führt zu eine Stichprobenanzahl von ca. 30000 Messwerten für die gesamte Fahrt.

\section{Messergebnisse}

Eine Messfahrt wurde am 20. August 2014 ab 11 Uhr durchgeführt. An diesem Tag kam es zu keinen besonderen Wetterereignissen und der 195Index zeigte für die Region Niedersachsen „sehr geringe ionosphärische Aktivitäten" an [10]. Somit ist nicht mit großen atmosphärischen Einflüssen auf das Messergebnis zu rechnen. Im Anschluss an die Messfahrt wurden die aufgezeichneten Frequenzbänder anschließend elfmal hintereinander im Labor abgespielt. Die Zahl 11 ist darauf zurückzuführen, dass anschließend das Gerät nicht mehr zur Verfügung stand.

\subsection{Positionsaktualität}

In einem ersten Schritt wurde die Positionsaktualität bestimmt. Die ermittelten Werte für den High-End und Low-Cost GNSS-Empfänger sind in Abb. 4 dargestellt. Es ist ersichtlich das der High-End Empfänger eine deutlich kleinere Positionsaktualität (mittlere Positionsaktualität: $30.8 \mathrm{~ms}, \mathrm{SD}(95 \%): 5.5 \mathrm{~ms}$ ) aufweist als der Low-Cost Empfänger(mittlere Positionsaktualität: $100.2 \mathrm{~ms}, \mathrm{SD}(95 \%): 11.5 \mathrm{~ms})$. 


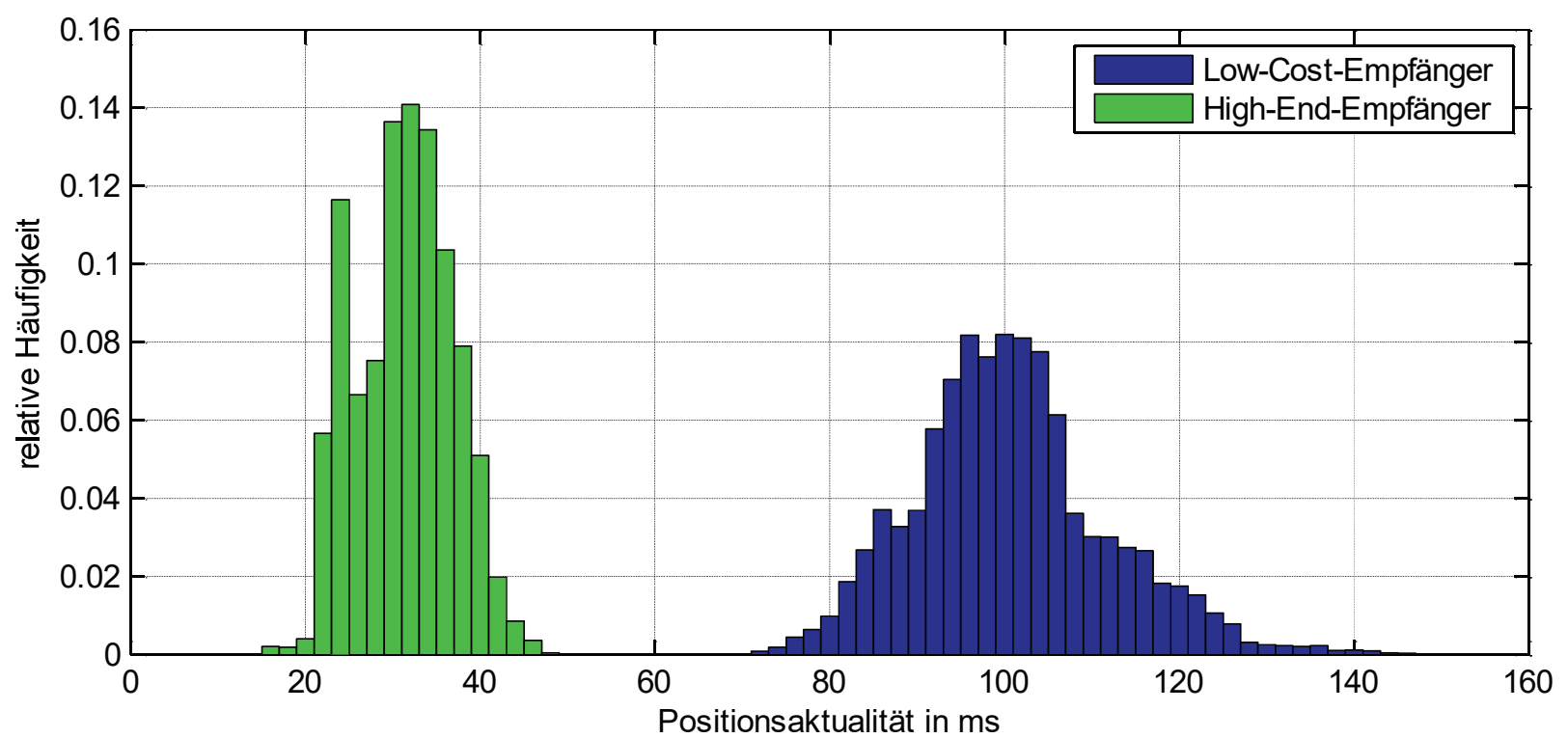

Abb. 4: Positionsaktualität für die gesamte Messfahrt für den Low-Cost und High-End GNSS-Empfänger

Als nächstes stellt sich die Frage inwieweit die Positionsaktualität über die einzelnen Prüfszenarien variiert. Die Positionsaktualitäten und Standardabweichungen sind in Tab. 1 aufgetragen. Es kann identifiziert werden, dass sowohl die Anzahl der getrackten Satelliten als auch der Grad der Herausforderung der Messbedingungen einen großen Einfluss auf die Positionsaktualität hat. So stechen die Großstadt Szenarien hervor. Die vermeintlich einfacheren Szenarien weisen eine geringere Positionsaktualität auf. Einzig Messfahrt Überland 1 folgt diesem Verhalten nicht. Dies ist darauf zurückzuführen, dass die Strecke Überland 1 entlang einer einseitigen Baumreihe geführt hat. Dies bedeutet für den Empfänger einen erhöhten Rechenaufwand, wenn einzelnen Satellitensignale permanent abgeschattet werden.

Als nächstes ist es interessant zu untersuchen, wie sich die Positionsaktualität auf die Messgenauigkeit auswirkt. Dafür werden zunächst die Referenzpositionen zu dem Zeitpunkt der Bereitstellung der Positionsdaten benötigt. Da das Referenzmesssystem nicht im $1000 \mathrm{~Hz}$ Takt arbeitet wird die Referenzposition durch das Interpolieren der nächsten Referenzwerte zu dem Zeitpunkt des Bereitstellung erzeugt.

Durch die Taktrate von $5 \mathrm{~Hz}$ des Referenzmesssystems und wegen der geringen Dynamik von Fahrzeugen im öffentlichen Straßenverkehr sind hier nur geringe Interpolationsfehler zu erwarten. In Abb. 5 sind die horizontale Ortsrichtigkeit mit und ohne Berücksichtigung der Positionsaktualität und die Geschwindigkeit des Fahrzeugs auf- getragen. Es wird ersichtlich, dass die horizontale Ortsrichtigkeit mit der Geschwindigkeit zunehmend an Bedeutung gewinnt. So wächst durch die Geschwindigkeit auf der Autobahn (um den Messwert $2.5 \cdot 10^{4}$ ) die Ortsrichtigkeit um ca. $3 \mathrm{~m}$ an, In anderen Prüfszenarien in denen die Geschwindigkeit geringer ist, ist der Einfluss der Positionsaktualität folglich gering.

\begin{tabular}{|c|c|c|c|}
\hline $\begin{array}{l}\text { 은 } \\
\frac{\mathbb{D}}{\leftarrow} \\
\stackrel{N}{N} \\
\text { N }\end{array}$ & 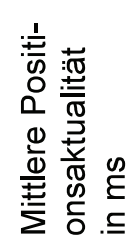 & 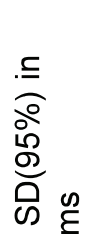 & 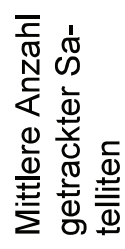 \\
\hline $\begin{array}{l}\text { Großstadt ohne } \\
\text { Bäume }\end{array}$ & 102.9 & 23.8 & 7.4 \\
\hline $\begin{array}{l}\text { Großstadt mit } \\
\text { Bäumen }\end{array}$ & 102.5 & 23.0 & 7.5 \\
\hline Stadt & 99.4 & 22.5 & 7.5 \\
\hline Überland 1 & 102.1 & 23.7 & 8.5 \\
\hline Überland 2 & 98.7 & 17.1 & 8.3 \\
\hline Waldfahrt & 96.7 & 22.2 & 7.1 \\
\hline Autobahnfahrt & 99.3 & 17.8 & 8.2 \\
\hline
\end{tabular}




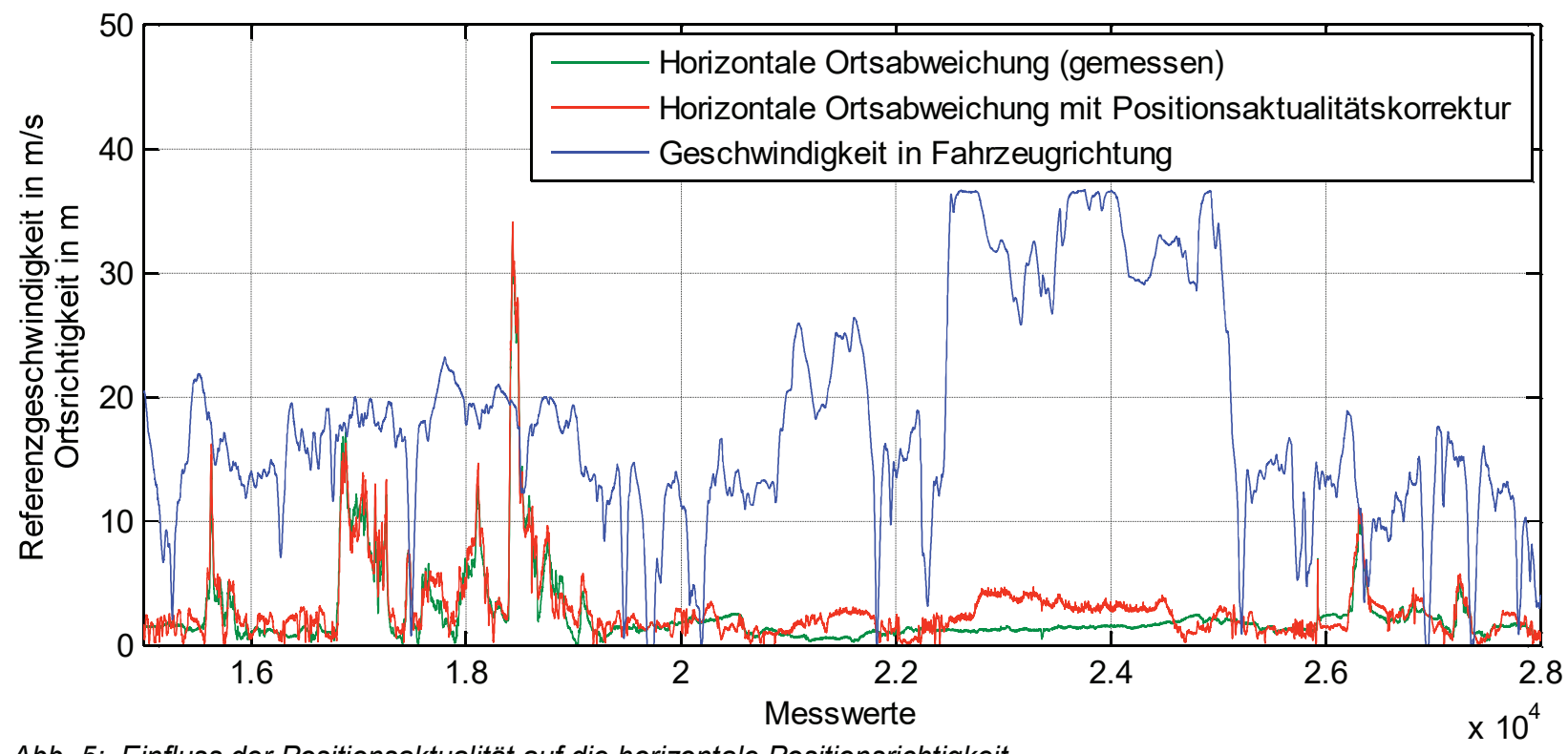

Abb. 5: Einfluss der Positionsaktualität auf die horizontale Positionsrichtigkeit

\subsection{Wiederholgenauigkeit}

Im Anschluss an die Positionsaktualität wird die Wiederholgenauigkeit untersucht. In Tab. 2 werden die Messergebnisse der Messungen dargestellt. Es ist ersichtlich, dass die Positionsrichtigkeit und die Positionspräzision der real aufgenommenen Daten von der Wiederholrichtigkeit und der Wiederholpräzision deutlich Abweichungen zeigen. Dabei ist jedoch keine Tendenz erkennbar. Der Zahlenwert der Positionsrichtigkeit steigt beispielsweise für die Szenarien Großstadt mit Bäumen und Überland 1 an, verringert sich jedoch auch bei Szenarien wie Großstadt und Waldfahrt. Durch dieses Verhalten lassen sich keine systematischen Fehler in dem Record-und-Replay Prozess identifizieren. Generell kann festgehalten werden, dass die Messergebnisse zeigen, dass eine Einzelfahrt nicht ausreicht um die Qualität eines GNSS-Empfängers zu ermitteln.

Für die Szenarien Waldfahrt und Großstadt konnten Messrichtigkeitsverbesserungen im Bereich von einigen Metern gezeigt werden. Die
Anzahl der eliminierten Ausreißer ist sehr gering. Sie liegt maximal im Bereich von 1.5\% der gesamt erhobenen Messwerte für die Szenarien. Dies ist deutlich unter dem Wert (maximaler Ausschluss von 2/9 der Messwerte) der für die Zertifizierung von Flächenmesssystemen vorgesehen ist [9].

\section{Zusammenfassung}

In diesem Beitrag wurde ein Prüfaufbau vorgestellt, der es ermöglicht, satellitenbasierte Ortungssysteme reproduzierbar und vergleichbar zu qualifizieren. Die aufgezeichneten Szenarien können anschließend wiederholt abgespielt und weitere Prüfgegenstände bei gleichen Bedingungen untersucht werden.

Der eingeführte und untersuchte Parameter der Positionsaktualität wurde für einen High-End und einen Low-Cost-Empfänger ermittelt. Es konnte gezeigt werden das der High-End Empfänger deutlich schnellere Positionsdaten liefert als der Low-Cost Empfänger.

Tab. 2: Messergebnisse der Wiederholrichtigkeit und Wiederholpräzision

\begin{tabular}{lcccccc}
\hline Szenario & $\begin{array}{c}\text { Positions- } \\
\text { richtigkeit } \\
\text { 2D in m }\end{array}$ & $\begin{array}{c}\text { Positions- } \\
\text { präzision } \\
\text { in m }\end{array}$ & $\begin{array}{c}\text { Wiederhol- } \\
\text { richtigkeit } \\
\text { in m }\end{array}$ & $\begin{array}{c}\text { Wiederhol- } \\
\text { präzision } \\
\text { in m }\end{array}$ & $\begin{array}{c}\text { Stichproben- } \\
\text { größe }\end{array}$ & Ausreißer \\
\hline Großstadt mit Bäumen & 6.17 & 4.21 & 6.98 & 5.84 & 19250 & 125 \\
Großstadt ohne Bäume & 5.02 & 9.41 & 3.25 & 2.29 & 22410 & 247 \\
Stadt 1 & 1.51 & 0.49 & 1.45 & 0.44 & 10109 & 0 \\
Überland 1 & 1.05 & 1.28 & 1.96 & 0.85 & 39664 & 47 \\
Überland 2 & 1.43 & 0.89 & 1.29 & 0.61 & 35464 & 0 \\
Autobahnfahrt & 1.27 & 0.79 & 1.34 & 0.37 & 29393 & 494 \\
Waldfahrt & 8.41 & 8.51 & 4.51 & 4.62 & 38962 & 518 \\
\hline
\end{tabular}


Darüber hinaus ermöglicht das wiederholte Abspielen der aufgezeichneten Satellitensignale eine umfassendere Untersuchung im Hinblick auf das stochastische Verhalten von GNSSEmpfängern. Es zeigte sich, dass für anspruchsvolle Szenarien das einfache Durchfahren oder Abspielen der Signale keine repräsentative Bestimmung der Qualitätsmerkmale ermöglicht. Vielmehr sind starke Unterschiede zwischen der Messfahrt und den Messergebnissen aus dem Playback zu erkennen. Eine Tendenz ist dabei nicht erkennbar.

\section{Literaturverzeichnis}

[1] AXIO-NeT GmBH:AXIO-NET PED : Der Präzise Echtzeitdienst mit einer Messgenauigkeit von $+/-2 \mathrm{~cm}$. URL http://www.axio-net.eu/produkte-loesungen/d/axio-net-ped/ - Überprüfungsdatum 2015-07-24.

[2] DEBIAO LU: GNSS for Train Localisation Performance Evaluation and Verification. Braunschweig, Technische Universität Braunschweig, Diss., 2014.

[3] Department OF DEFENCE: RTCA DO229:D: Minimum Operational Performance Standards for Global Positioning System/Wide Area Augmentation System Airborne Equipment. Washington D.C., 2006.

[4] DEUTSChES INSTITUT FÜR NORMUNG E.V.: DIN 2342:2011-08: Begriffe der Terminologielehre. Berlin, 2011.

[5] DEUTSCHES INSTITUT FÜR NORMUNG E.V.; INTERNATIONAL ORGANIZATION FOR STANDARDIZATION: DIN ISO 5725:2002-12: Genauigkeit (Richtigkeit und Präzision) von Messverfahren und Messergebnissen Teil 2 Grundlegende Methode für die Ermittlung der Wiederhol- und Vergleichspräzision eines vereinheitlichten Messverfahrens. Berlin, 2002.

[6] EUROPEAN COMMITTEE FOR STANDARDIZATION: EN 16803-1 (Draft): Use of GNSSbased positioning for road Intelligent Transport Systems (ITS) - Definitions and system engineering procedures for the establishment and assessment of performances. 2015.

[7] EUROPEAN TELECOMMUNICATIONS STANDARDS INSTITUTE: ETSI TS 101 593:201601: Satellite Earth Stations and Systems (SES); GNSS based location systems; Part 5: Performance Test Specification. Sophia Antipolis, Frankreich, 2012.

[8] INTERNATIONAL ELECTROTECHNICAL COMMISSION: IEC 61108:1: Maritime navigation and radiocommunication equipment and systems- Global navigation satellite -systems (GNSS)- Part 1: Global positioning system (GPS) - Receiver equipment Performance standards, methods of testing and required test results. Genf, Schweiz, 2010.

[9] JOINT RESEARCH CENTER; EUROPEAN COMISSION: JRC Technical Note 51300: Area measurement validation scheme. Luxembourg, 2009.

[10] LANDESAMT FÜR GEOINFORMATION UND LANDENTWICKLUNG NIEDERSACHSEN:IOnOsphärischer Index 195 vom 20.8.2014. URL http://www.Ignapp.niedersachsen.de/sapos/iono_index.htm - Überprüfungsdatum 2015-08-12.

[11] NORTH ATLANTIC TREATY ORgANIZATION: STANAG 4278:3: Method of Expressing Navigation Accuracies. Brussels, 1995.

[12] OXFORD TECHNOLOGIES:RT3000: Hochpräzise GPS gestützte Inertialmesssysteme Datenblatt. URL http://www.oxts.com/Downloads/Products/RT3000/RT3000_DE.pdf - Überprüfungsdatum 2015-07-23.

[13] SCHNIEDER, Lars: Formalisierte Terminologien technischer Systeme und ihrer Zuverlässigkeit. Braunschweig, Technische Universität Braunschweig, Diss., 2010.

[14] SpIEgel, Dirk ; BeCKER, Uwe:A latency and accuracy investigation of satellite receivers for automotive applications. In: Proceedings of the International Symposium on Certification of GNSS Systems \& Services - CERGAL 2015

[15] SPIEGEL, Dirk ; GRASSO TORO, Federico ; SCHNIEDER, Eckehard:A Satellite Independent High Dynamic Test Bed and First Measurement Results. In: Proceedings of the 27th International Technical Meeting of The Satellite Division of the Institute of Navigation (ION GNSS+ 2014) 2014, S. 433-439.

[16] SPIRENT:GSS6425 GNSS Multi-Frequency Record Playback System : Datasheet with Product Specification. 2014.

[17] Wegener, Marco: Über die metrologische Qualität der Fahrzeugortung. Braunschweig, Technische Universität Braunschweig, Diss., 2013. 